\title{
Orbit determination for next generation space clocks
}

\author{
L. Duchayne ${ }^{1}$, F. Mercier ${ }^{2}$, and P. Wolf ${ }^{1}$ \\ 1 LNE-SYRTE, Observatoire de Paris, CNRS, UPMC, 75014 Paris, France \\ e-mail: peter.wolf@obspm.fr \\ 2 Centre National d'Études Spatiales, France
}

Received 20 February 2008 / Accepted 26 April 2009

\section{ABSTRACT}

\begin{abstract}
We study the requirements on orbit determination compatible with operation of next generation space clocks at their expected uncertainty. Using the ACES (Atomic Clock Ensemble in Space) mission as an example, we develop a relativistic model for time and frequency transfer to investigate the effects of orbit determination errors. For the orbit error models considered we show that the required uncertainty goal can be reached with relatively modest constraints on the orbit determination of the space clock, which are significantly less stringent than expected from "naive" estimates. Our results are generic to all space clocks and represent a significant step towards the generalized use of next generation space clocks in fundamental physics, geodesy, and time/frequency metrology.
\end{abstract}

Key words. relativity - reference systems - time - ephemerides - instrumentation: miscellaneous - space vehicles: instruments

\section{Introduction}

Over the last decade of the 20th and the start of the 21 st century, the uncertainty of atomic clocks has decreased by over two orders of magnitude, improving from $10^{-14}$ to below $10^{-16}$, in relative frequency (Bize et al. 2005; Heavner et al. 2005; Oskay et al. 2006; Rosenband et al. 2008). This rapid evolution has been achieved by recent technological breakthroughs (laser cooling and trapping of atoms and ions), which allow effective control and reduction in the motion of the atoms and correspondingly long interrogation times. Atomic fountain microwave clocks use freely falling laser-cooled atoms and were the first to reach uncertainties below $10^{-15}$. Some of them achieving uncertainties in the low $10^{-16}$ range. They are now closely followed, and even surpassed, by trapped ion and neutral atom optical clocks, the best of which have uncertainties of below $10^{-16}$.

Space applications in e.g. fundamental physics, geodesy, time and frequency metrology, and navigation are among the most promising for this new generation of clocks. When onboard terrestrial or solar system satellites, their exceptional frequency stability and accuracy make them prime tools for testing the fundamental laws of nature, and studying the Earth's and solar system gravitational potentials and their evolution. In the longer term, they are likely to become the primary time reference for the Earth, because clocks on the ground will be affected by the poor knowledge of the geopotential on the surface (Wolf \& Petit 1995).

When comparing a clock on a low Earth orbiting satellite $(\approx 1000 \mathrm{~km}$ altitude) with one on the ground, one observes a difference of $\approx 10^{-10}$ in relative frequency due to the relativistic gravitational frequency shift. Measuring this difference with an uncertainty of $10^{-17}$ would allow a test of the gravitational frequency shift to a few parts in $10^{7}$ or equivalently, a determination of the potential difference between the clocks at the $10 \mathrm{~cm}$ level. The latter would contribute significantly to the knowledge of the geopotential and related applications in geophysics, representing the first realisation of relativistic geodesy
(Bjerhammar 1985; Soffel 1998) where the fundamental observable is directly the gravitational potential inferred from the relativistic redshift.

From the above, it is obvious that the next generation space clocks at the envisaged uncertainty level require a fully relativistic analysis and modelling, not only of the clocks (in space and on the ground) but also of the time/frequency transfer method used to compare them (Allan \& Ashby 1986; Klioner 1992; Petit \& Wolf 1994; Wolf \& Petit 1995; Blanchet et al. 2001; Linet \& Teyssandier 2002). A highly accurate space clock is indeed of limited use unless (1) it can be compared with ground clocks using a method that does not degrade the overall uncertainty, and (2) the behaviour of the clocks as a function of their positions and velocities can be modelled with sufficient accuracy. As an example, simple order of magnitude estimates of the relativistic gravitational frequency shift show that an $1 \mathrm{~m}$ error in the position of the clocks leads to an error of $\approx 10^{-16}$ in the determination of their frequency difference. Similarly when using a one-way system (GPS like) for the time transfer, a $1 \mathrm{~m}$ position error leads to an error of $\approx 3 \times 10^{-9}$ s in the synchronisation i.e., $\geq 10^{-14}$ in relative frequency over one day.

In this paper, we study in more detail the requirements for orbit determination compatible with operation of next generation space clocks at the required uncertainty, based on a completely relativistic model. We use the example of the ACES (atomic clock ensemble in space) mission, an ESA-CNES project to be installed onboard the ISS (International Space Station) in 2013. It consists of two atomic clocks and a two-way time transfer system (microwave link, MWL) with an overall uncertainty goal of 1 part in $10^{16}$ after a ten day integration (see Sect. 3 for more details). We show that the required accuracy can be reached with relatively modest constraints on the orbit determination of the space clock of $\approx 10 \mathrm{~m}$ in position (for the considered orbit error models), which is about an order of magnitude less stringent than expected from "naïve" estimates ( $\approx 1 \mathrm{~m}$, see above). This is because of the first order cancellation between the velocity and position part of the orbit determination error in the determination 
of the relativistic frequency shift of the space clock, and the use of a two-way time transfer system (MWL) leading to first order cancellation of the position errors in the clock comparison (see Sect. 6). Our results are applicable to all space clocks (not only to the ACES mission) and represent a significant step towards the generalised use of next generation space clocks in fundamental physics, geodesy, and time/frequency metrology, since they show that the constraints on the orbit determination of the space clock are significantly less stringent than previously thought.

In Sects. 2 and 3, we briefly describe the ACES mission and the relativistic model used for the clocks and the time transfer. We explicitly derive the effect of orbit errors on the clock comparison in Sect. 4. Up to this point, our results are completely general (within the specified approximations) with no assumptions about the expected orbit determination errors. We then apply those results using two examples of expected orbit errors (Sect. 5). Our main results are the calculation of the effect of these orbit errors on the determination of the relativistic frequency shift of the clocks and on the time transfer (MWL) for the ACES mission (Sect. 6). We provide the overall requirements of orbit determination for the ACES mission, and show that the mission objectives can be achieved with relatively modest accuracy of orbit determination. We discuss those results and conclude in Sect. 7.

\section{The ACES mission}

Led by CNES and ESA, the ACES project aims to establish several highly stable clocks in 2013 on the ISS. The ACES payload includes two clocks, a hydrogen maser (SHM developed by TEMEX), and a cold atom clock PHARAO (developed by CNES) respectively for short and long term performances, respectively, and a microwave link for communication and time/frequency comparison. The frequency stability of PHARAO onboard the ISS is expected to be better than $10^{-13}$ for one second, $3 \times 10^{-16}$ over one day, and $1 \times 10^{-16}$ over ten days, with an accuracy goal of $1 \times 10^{-16}$ in relative frequency.

The ACES mission has as objectives:

- to operate a cold atom clock in microgravity with a $100 \mathrm{mHz}$ linewidth;

- to compare the high performances of the two atomic clocks in space (PHARAO and SHM) and achieve a stability of $3 \times$ $10^{-16}$ over one day;

- to perform time comparisons between the two space clocks and ground clocks;

- to carry out tests of fundamental physics such as a gravitational redshift measurement and a test of Lorentz invariance, and to search for a possible drift in the fine structure constant $\alpha$;

- to perform precise measurements of the Total Electron Content (TEC) in the ionosphere, the tropospheric delay and the Newtonian potential.

The time transfer is performed using a microwave two-way system, called micro-wave link (MWL). An additional frequency is added to measure and correct the ionospheric delay to the required level. It uses carriers of frequency 13.5, 14.7, and $2.25 \mathrm{GHz}$, modulated by pseudo random codes respectively at $10^{8} \mathrm{~s}^{-1}, 10^{8} \mathrm{~s}^{-1}$, and $10^{6} \mathrm{~s}^{-1}$ chip rates. It has also four channels that allow four ground stations to be compared with the ISS clock at the same time.

According to the mission specifications, the microwave link must synchronize two atomic clocks with a time stability of $\leq 0.3$ ps over $300 \mathrm{~s}, \leq 7$ ps over one day, and $\leq 23$ ps over 10 days.
The performance of this link is a key issue because it must perform high precision time comparisons without damaging the high performances of the clocks.

For our purposes, we express the above requirements for the MWL in a simplified form by the temporal Allan deviation $\left(\sigma_{x}(\tau)\right)$ expressed in seconds, given by:

$\sigma_{x}(\tau)=5.2 \times 10^{-12} \mathrm{~s} \cdot \tau^{-\frac{1}{2}}$

where the integration time $\tau$ is in seconds. Equation (1) is valid for a single satellite pass over a ground station (for integration times $\tau$ lower than $300 \mathrm{~s}$ ). For longer integration times,

$\sigma_{x}(\tau)=2.4 \times 10^{-14} \mathrm{~s} \cdot \tau^{\frac{1}{2}}$

The temporal Allan deviation can be related to frequency instability in terms of the modified Allan deviation $\operatorname{Mod}_{y}(\tau)$ given by

$\operatorname{Mod}_{y}(\tau)=\frac{\sqrt{3} \sigma_{x}(\tau)}{\tau}$

We assume that (1) and (2) represent our upper limits to the calculation of all perturbing effects in the following sections, together with the overall accuracy requirement (maximum allowed frequency bias) of $1 \times 10^{-16}$ in relative frequency.

\section{Relativistic model for clocks and time transfer of ACES}

In a general relativistic framework, each clock produces its own local proper time, in our case $\tau^{\mathrm{g}}$ and $\tau^{\mathrm{s}}$ for ground and space clocks, respectively.

To model signal propagation between the ground and the space stations, we use a non-rotating geocentric space-time coordinate system. Thus, $t=x_{0} / c$ is the geocentric coordinate time, and $\boldsymbol{x}=\left(x_{1}, x_{2}, x_{3}\right)$ are the spatial coordinates, where $c$ is the speed of light in vacuum $\left(c=299792458 \mathrm{~m} \mathrm{~s}^{-1}\right)$. We denote $U(t, \boldsymbol{x})$ as the total Newtonian potential at the coordinate time $t$ and the position $x$ with the convention that $U \geq 0$ (Soffel et al. 2003). In these coordinates, the metric is given by an approximate solution of Einstein's equations that is valid for low velocity and potential $\left(\frac{U}{c^{2}} \ll 1\right.$ and $\left.\frac{v^{2}}{c^{2}} \ll 1\right)$ :

$\mathrm{d} s^{2}=-\left(1-\frac{2 U(t, \boldsymbol{x})}{c^{2}}\right) c^{2} \mathrm{~d} t^{2}+\left(1+\frac{2 U(t, \boldsymbol{x})}{c^{2}}\right) \mathrm{d} \boldsymbol{x}^{2}$,

where higher order terms can be neglected for our purposes (Wolf \& Petit 1995).

In this system, each emission or reception event (at the antenna phase center) is identified by its coordinate time $t_{i}$ (Fig. 1) and a coordinate time interval is defined by $T_{i j}=t_{j}-t_{i}$. We define $\boldsymbol{x}_{\mathrm{g}}, \boldsymbol{v}_{\mathrm{g}}$, and $\boldsymbol{a}_{\mathrm{g}}$ to be the position, velocity, and acceleration of the ground station, respectively, and $\boldsymbol{x}_{\mathrm{s}}, \boldsymbol{v}_{\mathrm{s}}$, and $\boldsymbol{a}_{\mathrm{s}}$ to be the position, velocity, and acceleration of the space station, respectively.

The ACES mission uses two different antennas: one $K u$-band antenna for uplink and downlink, and one $S$-band antenna for downward signals only. The antennae are characterized by their phase pattern, which describes the position of the antenna phase center as a function of the direction of the incoming signal. The phase patterns of the MWL antennas have been measured in the laboratory at all three frequencies. For example, the phase variations with direction of the $\mathrm{Ku}$-band antenna can reach up to $\sim 0.1 \mathrm{rad}(\sim 1 \mathrm{ps}$ in time). Those variations can be corrected from 


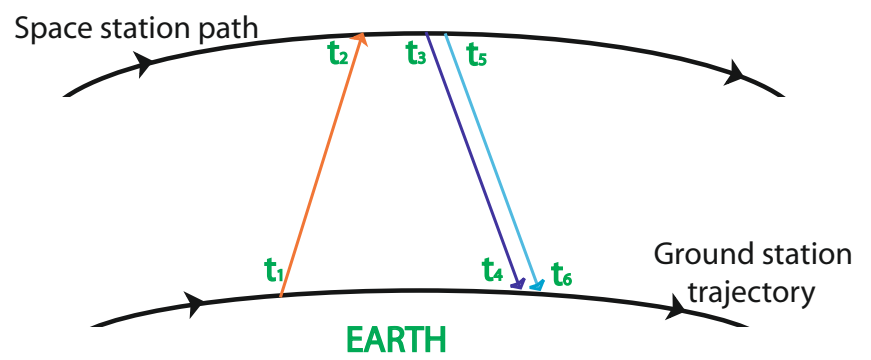

Fig. 1. MWL principle.

the known phase pattern and a knowledge of ISS attitude (i.e., direction of the incoming signal). Once corrected the remaining errors, mostly due to uncertainties in ISS attitude, are below the MWL specifications.

The $f_{1}$ frequency signal is emitted by the ground station at the coordinate time $t_{1}$ and received by the space station at $t_{2}$. The $f_{2}$ and $f_{3}$ frequency signals are emitted from the space station at $t_{3}$ and $t_{5}$, and received at the ground station at $t_{4}$ and $t_{6}$. The third frequency is added to measure the TEC in the ionosphere, which allows the correction of the ionospheric delay.

The MWL is characterized by its continuous emission. It measures the time offsets between the locally generated and received signals. It provides three measurements (or observables) of the code (one on the space station, two on the ground) and three measurements of the phase of the carrier frequency at a sampling rate of one Hertz.

An observable is related to the phase comparison between a signal derived from the local oscillator and the received signal, corrected for the frequency difference mainly due to the first order Doppler effect (see Bahder 2003, for details of a similar procedure used in GPS). If we consider a particular bit of the signal that is produced locally at $\tau_{\mathrm{p}}$ and received at $\tau_{\mathrm{a}}$, an observable is a measurement of the local proper time interval between these two events. The true measurement of the phase difference $\Delta \Phi\left(\tau_{\mathrm{a}}, \boldsymbol{x}_{\mathrm{a}}\right)$ occurs at the single space-time point $\left(\tau_{\mathrm{a}}, \boldsymbol{x}_{\mathrm{a}}\right)$ and is labeled with the arrival proper time $\tau_{\mathrm{a}}$. It can be expressed as

$\Delta \tau\left(\tau_{\mathrm{a}}, \boldsymbol{x}_{\mathrm{a}}\right)=\frac{\Delta \Phi\left(\tau_{\mathrm{a}}, \boldsymbol{x}_{\mathrm{a}}\right)}{\omega}+\delta \tau=\tau_{\mathrm{p}}-\tau_{\mathrm{a}}$

where $\omega$ is a conversion factor from phase to proper time depending on the nominal carrier and code frequencies, and $\delta \tau$ represents the measurement errors (e.g., difference between the clock and ideal proper time and measurement phase noise).

Considering the experimental uncertainties in the ACES mission (see Eqs. (1) and (2)), we neglect any terms in the relativistic model that, when maximised, lead to corrections of less than $3 \times 10^{-13} \mathrm{~s}$ in time. Numerical applications are necessary to evaluate which terms can be neglected. For this purpose, we consider the International Space Station with a circular trajectory at an altitude of $400 \mathrm{~km}$ in a plane inclined by $51.6^{\circ}$ with respect to the equatorial plane. Its velocity is $v_{\mathrm{s}}=7.7 \times 10^{3} \mathrm{~m} \mathrm{~s}^{-1}$ in a gravitational potential of $U_{\mathrm{s}} / c^{2}=6.5 \times 10^{-10}$. The ground station has a velocity $v_{\mathrm{g}}=465 \mathrm{~m} \mathrm{~s}^{-1}$ at a gravitational potential of $U_{\mathrm{g}} / c^{2}=6.9 \times 10^{-10}$.

The tropospheric delay $\Delta^{\text {tropo }}$ is considered to be independent of the signal frequency, and slowly varying in time with an amplitude of less than 100 ns.

We assume that the density of electrons in the ionosphere $N_{\mathrm{e}}$ is lower than $2 \times 10^{12}$ electrons $/ \mathrm{m}^{3}$. The ionospheric delay $\Delta^{\text {iono }}$ is maximum for the $f_{3}$ frequency signal with an amplitude of less than $10 \mathrm{~ns}$.
The relation between the proper time $\tau$ and the coordinate time $t$ is given to sufficient accuracy by

$\frac{\mathrm{d} \tau}{\mathrm{d} t}=1-\left(\frac{U(t, \boldsymbol{x})}{c^{2}}+\frac{v^{2}(t)}{2 c^{2}}\right)+O\left(c^{-4}\right)$.

Higher order terms of Eq. (6) have negligible effects at the projected uncertainty of $1 \times 10^{-16}$ in relative frequency of the ACES clocks (Wolf \& Petit 1995). We note however, that some care must be taken when evaluating the Newtonian potential $U(t, \boldsymbol{x})$ in (6) for the ground or space clock (Wolf \& Petit 1995).

The ACES mission aims to measure the variation in the desynchronisation between ground and space clocks with time, that is to say, the function $\tau^{\mathrm{g}}(t)-\tau^{\mathrm{s}}(t)$. It is evaluated by combining the measurements performed on the ground and onboard the space station and a precise calculation of the signal propagation times. To be able to control $T_{23}$ (see below), we combine two measurements $\Delta \tau^{\mathrm{s}}\left(\tau^{\mathrm{s}}\left(t_{2}\right)\right)$ and $\Delta \tau^{\mathrm{g}}\left(\tau^{\mathrm{g}}\left(t_{4}\right)\right)$ but with $\tau^{\mathrm{s}}\left(t_{2}\right) \neq \tau^{\mathrm{g}}\left(t_{4}\right)$. The expression of desynchronisation then reads (see Duchayne 2008, for a detailed derivation)

$$
\begin{aligned}
\tau^{\mathrm{g}}\left(t_{\mathrm{a}}\right)-\tau^{\mathrm{s}}\left(t_{\mathrm{a}}\right)= & \frac{1}{2}\left(\Delta \tau^{\mathrm{s}}\left(\tau^{\mathrm{s}}\left(t_{2}\right)\right)-\Delta \tau^{\mathrm{g}}\left(\tau^{\mathrm{g}}\left(t_{4}\right)\right)\right. \\
& +T_{12}-T_{34}-\int_{t_{1}}^{t_{2}}\left(\frac{U\left(t, \boldsymbol{x}_{\mathrm{g}}\right)}{c^{2}}+\frac{v_{\mathrm{g}}^{2}(t)}{2 c^{2}}\right) \mathrm{d} t \\
& \left.+\int_{t_{3}}^{t_{4}}\left(\frac{U\left(t, \boldsymbol{x}_{\mathrm{s}}\right)}{c^{2}}+\frac{v_{\mathrm{s}}^{2}(t)}{2 c^{2}}\right) \mathrm{d} t\right),
\end{aligned}
$$

where $t_{\mathrm{a}}=\frac{t_{2}+t_{4}}{2}$, and $\Delta \tau^{\mathrm{s}}\left(\tau^{\mathrm{s}}\left(t_{2}\right)\right)$ and $\Delta \tau^{\mathrm{g}}\left(\tau^{\mathrm{g}}\left(t_{4}\right)\right)$ are the observables from the ground and onboard the satellite at the coordinate times $t_{2}$ and $t_{4}$, respectively, and where we have neglected nonlinearities in $\tau^{\mathrm{g}}(t)$ and $\tau^{\mathrm{s}}(t)$ over the interval $t_{4}-t_{2}$ (few milliseconds). The integral terms result from proper time to coordinate time transformations. They are small corrections of order $10^{-12}$ $\mathrm{s}$ to the desynchronisation $\tau^{\mathrm{g}}\left(t_{\mathrm{a}}\right)-\tau^{\mathrm{s}}\left(t_{\mathrm{a}}\right)$.

However, it is the derivative with respect to the coordinate time $t$ of Eq. (7) that has to be studied for applications such as the gravitational redshift test or geodesy, and then compared with the relation derived from Eq. (6):

$$
\begin{aligned}
\frac{\mathrm{d} \tau^{\mathrm{g}}}{\mathrm{d} t}-\frac{\mathrm{d} \tau^{\mathrm{s}}}{\mathrm{d} t}= & \frac{1}{c^{2}} \cdot\left(U\left(t, \boldsymbol{x}_{\mathrm{s}}\right)-U\left(t, \boldsymbol{x}_{\mathrm{g}}\right)\right. \\
& \left.+\frac{v_{\mathrm{s}}^{2}(t)}{2}-\frac{v_{\mathrm{g}}^{2}(t)}{2}\right)+O\left(c^{-4}\right) .
\end{aligned}
$$

We note that any constant term appearing in the desynchronisation given by Eq. (7) will have no effect on the final result in Eq. (8) because of the derivation.

In (7), the difference $T_{12}-T_{34}$ needs to be calculated from the knowledge of satellite and ground positions and velocities (orbit determination). For example, $T_{12}$ is the time interval elapsed between emission from the ground station and reception by the satellite of the $f_{1}$ frequency signal. It can be written as

$$
\begin{aligned}
T_{12}= & \frac{R_{12}}{c}+\frac{2 G M_{\mathrm{E}}}{c^{3}} \ln \left(\frac{x_{\mathrm{g}}\left(t_{1}\right)+x_{\mathrm{s}}\left(t_{2}\right)+R_{12}}{x_{\mathrm{g}}\left(t_{1}\right)+x_{\mathrm{s}}\left(t_{2}\right)-R_{12}}\right) \\
& +\Delta_{12}^{\text {tropo }}+\Delta_{12}^{\text {iono }}+O\left(\frac{1}{c^{4}}\right)
\end{aligned}
$$

where $R_{12}=\left\|\boldsymbol{R}_{12}\right\|=\left\|\boldsymbol{x}_{\mathrm{s}}\left(t_{2}\right)-\boldsymbol{x}_{\mathrm{g}}\left(t_{1}\right)\right\|$, the logarithmic term represents the Shapiro time delay (Shapiro 1964) (see e.g., Blanchet et al. 2001, for a detailed derivation), and where $\Delta_{12}^{\text {tropo }}$ and $\Delta_{12}^{\text {iono }}$ 
are the tropospheric and ionospheric delays along the signal path, respectively.

Only one of the clocks (which we assume here to be the ground clock) has a known relation with coordinate time $t$, so $\boldsymbol{x}_{\mathrm{s}}\left(t_{2}\right)$ cannot be directly obtained from the orbit determination, only $\boldsymbol{x}_{\mathrm{s}}\left(t_{1}\right)$ is known. Equation (9) is then modified to

$$
\begin{aligned}
T_{12}= & \frac{D\left(t_{1}\right)}{c}+\frac{\boldsymbol{D}\left(t_{1}\right) \cdot \boldsymbol{v}_{\mathrm{s}}\left(t_{1}\right)}{c^{2}} \\
& +\frac{D\left(t_{1}\right)}{2 c^{3}}\left(\left\|\boldsymbol{v}_{\mathrm{s}}\left(t_{1}\right)\right\|^{2}+\boldsymbol{D}\left(t_{1}\right) \cdot \boldsymbol{a}_{\mathrm{s}}\left(t_{1}\right)\right. \\
& \left.+\left(\frac{\boldsymbol{D}\left(t_{1}\right) \cdot \boldsymbol{v}_{\mathrm{s}}\left(t_{1}\right)}{D\left(t_{1}\right)}\right)^{2}\right) \\
& +\frac{2 G M_{\mathrm{E}}}{c^{3}} \ln \left(\frac{x_{\mathrm{g}}\left(t_{1}\right)+x_{\mathrm{s}}\left(t_{1}\right)+D\left(t_{1}\right)}{x_{\mathrm{g}}\left(t_{1}\right)+x_{\mathrm{s}}\left(t_{1}\right)-D\left(t_{1}\right)}\right) \\
& +\Delta_{12}^{\text {iono }}+\Delta_{12}^{\text {tropo }}+O\left(c^{-4}\right),
\end{aligned}
$$

where $\boldsymbol{D}(t)=\boldsymbol{x}_{\mathrm{s}}(t)-\boldsymbol{x}_{\mathrm{g}}(t), D(t)=\|\boldsymbol{D}(t)\|$, and where the unknown position $\boldsymbol{x}_{\mathrm{s}}\left(t_{2}\right)$ in Eq. (9) was expanded in a Taylor series around the corresponding known position $\boldsymbol{x}_{\mathrm{s}}\left(t_{1}\right)$. The ionospheric and tropospheric terms are related to the signal paths. They need to be calculated for $\boldsymbol{R}(t)$, but only $\boldsymbol{D}(t)$ is known. The corresponding correction is related to the motion of the space station during the atmospheric delay of the signal (the atmospheric equivalent of the "Sagnac" type term (2nd term in Eq. (10)). The corresponding corrections to both the tropospheric term and the ionospheric terms at frequencies $f_{1}$ and $f_{2}$ are negligible, but they are included for the $f_{3}$ signal (see Eq. (14)).

Assuming that $T_{23} \leq 10^{-3} \mathrm{~s}$, the expression of $T_{12}-T_{34}$, where all terms greater than $3 \times 10^{-13} \mathrm{~s}$ have been included when maximized for ACES can be evaluated by:

$$
\begin{aligned}
T_{12}-T_{34}= & 2 \frac{\boldsymbol{D}\left(t_{4}\right) \cdot \boldsymbol{v}_{\mathrm{g}}\left(t_{4}\right)}{c^{2}}+\frac{\boldsymbol{D}\left(t_{4}\right) \cdot \boldsymbol{\Delta} \boldsymbol{v}\left(t_{4}\right)}{c \cdot D\left(t_{4}\right)} T_{23} \\
& +2 \frac{D\left(t_{4}\right)}{c^{3}} \cdot\left(\boldsymbol{\Delta} \boldsymbol{v}\left(t_{4}\right) \cdot \boldsymbol{v}_{\mathrm{s}}\left(t_{4}\right)\right. \\
& \left.-\boldsymbol{D}\left(t_{4}\right) \cdot \boldsymbol{a}_{\mathrm{g}}\left(t_{4}\right)+\left\|\boldsymbol{\Delta} \boldsymbol{v}\left(t_{4}\right)\right\|^{2}\right) \\
& +\frac{T_{23}}{c^{2}} \cdot\left(\boldsymbol{\Delta} \boldsymbol{v}\left(t_{4}\right) \cdot \boldsymbol{v}_{\mathrm{s}}\left(t_{4}\right)\right. \\
& -\boldsymbol{D}\left(t_{4}\right) \cdot \boldsymbol{a}_{\mathrm{s}}\left(t_{4}\right)+2\left\|\boldsymbol{\Delta} \boldsymbol{v}\left(t_{4}\right)\right\|^{2} \\
& \left.-2 \boldsymbol{D}\left(t_{4}\right) \cdot \boldsymbol{\Delta} \boldsymbol{a}\left(t_{4}\right)-\left(\frac{\boldsymbol{D}\left(t_{4}\right) \cdot \boldsymbol{\Delta v}\left(t_{4}\right)}{D\left(t_{4}\right)}\right)^{2}\right) \\
& +\frac{T_{23}^{2}}{2 c D\left(t_{4}\right)}\left(\left\|\boldsymbol{\Delta} \boldsymbol{v}\left(t_{4}\right)\right\|^{2}-\boldsymbol{D}\left(t_{4}\right) \cdot \boldsymbol{\Delta} \boldsymbol{a}\left(t_{4}\right)\right. \\
& \left.-\left(\frac{\boldsymbol{D}\left(t_{4}\right) \cdot \boldsymbol{\Delta} \boldsymbol{v}\left(t_{4}\right)}{D\left(t_{4}\right)}\right)^{2}\right) \\
& +\Delta_{12}^{\text {iono }}-\Delta_{34}^{\text {iono }}+O\left(\frac{1}{c^{4}}\right),
\end{aligned}
$$

where $\Delta \boldsymbol{v}(t)=\boldsymbol{v}_{\mathrm{g}}(t)-\boldsymbol{v}_{\mathrm{s}}(t)$ and $\boldsymbol{\Delta} \boldsymbol{a}(t)=\boldsymbol{a}_{\mathrm{g}}(t)-\boldsymbol{a}_{\mathrm{s}}(t)$.

To obtain Eq. (11) we applied Eq. (10) to the upward and downward signals and expanded all positions and velocities in Taylor series around their values at $t_{4}$, which can be obtained from the time of measurement ("label" of the observable $\Delta \tau^{\mathrm{g}}\left(\tau^{\mathrm{g}}\left(t_{4}\right)\right)$ in Eq. (7)), on the ground.
The difference $T_{12}-T_{34}$ of upward and downward signals at $f_{1}$ and $f_{2}$ allows us to eliminate, to first order, delaying and restraining factors such as the range $(D / c)$, and troposphere or Shapiro effects. Because of the asymmetry of the paths, that cancellation is not perfect, and some terms remain (Eq. (11)) that depend on orbit determination as well as on the coordinate time interval $T_{23}$ elapsed between reception and emission at the phase center of the MWL antenna onboard the ISS.

The time interval $T_{23}$ can be controlled by "shifting" the time of the measurements on the ground or onboard the space station (e.g., shifting $\tau^{\mathrm{s}}\left(t_{2}\right)$ in $\Delta \tau^{\mathrm{s}}\left(\tau^{\mathrm{s}}\left(t_{2}\right)\right)$ of Eq. (7)) when postprocessing the data. This allows the control of the difference between emission and reception at the clock. However, $T_{23}$ given in Eq. (11) is defined at the antenna phase center, and therefore its control requires the knowledge (calibration and in situ measurement) of the instrumental delays (e.g., cables and electronics) in the MWL space segment. So the control and knowledge (uncertainty $\delta T_{23}$ ) of $T_{23}$ is determined by the uncertainty in the calibration and measurement of internal delays, which also play a role in the following.

The ionospheric effect $\Delta^{\text {iono }}$ on a radio signal of frequency $f$ can be modelled by (Bassiri \& Hajj 1993):

$$
\begin{aligned}
\Delta^{\text {iono }}= & \frac{40 \cdot 3082}{c \cdot f^{2}} \int N_{\mathrm{e}} \mathrm{d} L \\
& -\frac{7527}{f^{3}} \int\left(\boldsymbol{B}_{0} \cdot \boldsymbol{k}\right) \cdot N_{\mathrm{e}} \mathrm{d} L+O\left(\frac{1}{c^{4}}\right),
\end{aligned}
$$

where $\boldsymbol{B}_{0}$ is the Earth's magnetic field $\left(\left\|\boldsymbol{B}_{0}\right\| \approx 3.12 \times 10^{-5} \mathrm{~T}\right)$ and $\boldsymbol{k}$ is the direction of signal propagation.

The electron density integrated along the signal trajectory, $\int N_{\mathrm{e}} \mathrm{d} L$, is usually referred to as the total electron content (TEC). The difference in the ionospheric delays in Eq. (11) is then

$$
\begin{aligned}
\Delta_{12}^{\text {iono }}-\Delta_{34}^{\text {iono }}= & \left(\frac{1}{f_{1}^{2}}-\frac{1}{f_{2}^{2}}\right) \frac{40 \cdot 3082}{c} T E C \\
& -\frac{7527}{f_{1}^{3}} \int \frac{\boldsymbol{B}_{0} \cdot \boldsymbol{D}\left(t_{2}\right)}{D\left(t_{2}\right)} N_{\mathrm{e}} \mathrm{d} L \\
& -\frac{7527}{f_{2}^{3}} \int \frac{\boldsymbol{B}_{0} \cdot \boldsymbol{D}\left(t_{4}\right)}{D\left(t_{4}\right)} N_{\mathrm{e}} \mathrm{d} L+O\left(\frac{1}{c^{4}}\right)
\end{aligned}
$$

The second and third term in Eq. (13) represent the effect of the Earth's magnetic field and reach at most 0.5 ps for ACES, which is almost negligible. Therefore, an approximate estimation of these terms is sufficient.

The TEC is determined by combining the two downward signal observables. The time interval $T_{46}$ between the reception of the two signals depends on the difference in their internal delays at the emission from the space station (i.e., $T_{35}$ ) and the difference in their propagation time. Assuming that $T_{46}<1 \mu \mathrm{s}$, the expression of the Total Electron Content is then given by:

$$
\begin{aligned}
T E C= & \frac{c}{40.3082} \frac{1}{\frac{f_{2}^{2}-f_{3}^{2}}{f_{2}^{2} f_{3}^{2}}+\frac{f_{2}^{3}-f_{3}^{3}}{f_{2}^{3} f_{3}^{3}} \frac{7527 \cdot c}{40 \cdot 3} \frac{\boldsymbol{B}_{0} \cdot \boldsymbol{D}\left(t_{4}\right)}{D\left(t_{4}\right)}} \\
& \times\left(\Delta \tau^{\mathrm{g}}\left(\tau^{\mathrm{g}}\left(t_{4}\right)\right)-\Delta \tau^{\mathrm{g}}\left(\tau^{\mathrm{g}}\left(t_{6}\right)\right)\right. \\
& \left.+\frac{\boldsymbol{D}\left(t_{4}\right) \cdot \Delta \boldsymbol{v}\left(t_{4}\right)}{D\left(t_{4}\right)} \frac{T_{46}}{c}\right) /(1- \\
& \left.\frac{\boldsymbol{D}\left(t_{4}\right) \cdot \boldsymbol{v}_{\mathrm{s}}\left(t_{4}\right)}{D\left(t_{4}\right) \cdot c}\right)+O\left(\frac{1}{c^{4}}\right)
\end{aligned}
$$


where the final term in Eq. (14) is negligible when used for the time transfer (when inserted into Eq. (13)) but may be significant for the study of the TEC itself.

In summary, a reliable orbit determination is required for two main reasons: to calculate precisely the corrections in Eq. (11); and to evaluate correctly the terms on the right-hand side of Eq. (8) i.e., the second order Doppler and gravitational redshifts.

We also require a precise knowledge of the time interval $T_{23}$, (i.e., of the onboard internal delays) to be able to calculate the corresponding terms in Eq. (11) with sufficient accuracy.

In this work, we aim to estimate using simple orbit error models, which levels of uncertainty in orbit determination and calibration of internal delays (knowledge of $T_{23}$ ) are required to reach the required performances. For that purpose only the leading terms in Eq. (11) are required i.e.,

$$
\begin{aligned}
T_{12}-T_{34}= & 2 \frac{\boldsymbol{D}\left(t_{4}\right) \cdot \boldsymbol{v}_{\mathrm{g}}\left(t_{4}\right)}{c^{2}} \\
& +\frac{\boldsymbol{D}\left(t_{4}\right) \cdot \boldsymbol{\Delta v}\left(t_{4}\right)}{c \cdot D\left(t_{4}\right)} T_{23}+O\left(\frac{1}{c^{3}}\right),
\end{aligned}
$$

which, together with Eq. (8) for the relativistic frequency shift, is sufficient to derive the maximum allowed uncertainties in orbit determination and calibration of internal delays in order to stay below the limits given by Eqs. (1) and (2).

\section{Effects of orbit errors on clock comparison}

We now use Eqs. (15) and (8) to express the effects of station trajectory and time calibration uncertainties on both the time transfer and the relativistic frequency shift. In this section we make no assumptions about the orbit determination errors, our results being completely general and valid up to the neglected terms as described below.

We use $\left(\boldsymbol{X}_{\mathrm{a}}(t), \boldsymbol{V}_{\mathrm{a}}(t)\right)$ and $\left(\boldsymbol{X}_{\mathrm{a}}^{\prime}(t), \boldsymbol{V}_{\mathrm{a}}^{\prime}(t)\right)$ for the true and computed trajectories of the antenna phase center, respectively, and $\left(\boldsymbol{X}_{\mathrm{c}}(t), \boldsymbol{V}_{\mathrm{c}}(t)\right)$ and $\left(\boldsymbol{X}_{\mathrm{c}}^{\prime}(t), \boldsymbol{V}_{\mathrm{c}}^{\prime}(t)\right)$ for the true and computed trajectories of the clock reference point, respectively. We also define $\left(\boldsymbol{X}_{\mathrm{o}}(t), \boldsymbol{V}_{\mathrm{o}}(t)\right)$ the true trajectory and the true velocity of the center of mass of the ISS. These five trajectories are expressed in the non-rotating geocentric frame (GCRS, Geocentric Celestial Reference System) (Soffel et al. 2003).

On the one hand, the error in the time transfer is related to the uncertainties in $T_{12}-T_{34}$, and can be obtained from the simplified Eq. (15). It is dependent on the ground and space station trajectory knowledge, and on both the value of $T_{23}$ and its uncertainty $\delta T_{23}$. As said before, a precise knowledge of the time interval $T_{23}$ is related to the internal delay calibrations. The error in $T_{12}-T_{34}$ is

$$
\begin{aligned}
\delta\left(T_{12}-T_{34}\right)= & 2 \frac{\delta \boldsymbol{D} \cdot \boldsymbol{v}_{\mathrm{g}}+\boldsymbol{D} \cdot \delta \boldsymbol{v}_{\mathrm{g}}}{c^{2}}+\frac{\boldsymbol{D} \cdot \Delta \boldsymbol{v}}{c \cdot D} \delta T_{23} \\
& +\left(\frac{\delta \boldsymbol{D} \cdot \Delta \boldsymbol{v}}{c \cdot D}+\frac{\boldsymbol{D} \cdot \delta \Delta \boldsymbol{v}}{c \cdot D}\right. \\
& \left.-\frac{\boldsymbol{D} \cdot \Delta \boldsymbol{v}}{c \cdot D} \frac{\delta D}{D}\right) T_{23} .
\end{aligned}
$$

In general, ground station uncertainties are below $10 \mathrm{~cm}$. Thus, the uncertainty in the ground station position is negligible with respect to the ISS position errors and the knowledge of the vector $\boldsymbol{D}$ is only related to the uncertainty in the space station reference point, which is the antenna phase center. We then have $\delta \boldsymbol{D}=$ $\overrightarrow{\boldsymbol{X}_{\mathrm{a}} \boldsymbol{X}_{\mathrm{a}}^{\prime}}$, using the notation $\overrightarrow{\boldsymbol{X}_{i} \boldsymbol{X}_{j}} \equiv \boldsymbol{X}_{j}-\boldsymbol{X}_{i}$.
Equation (16) can then be written as:

$$
\begin{aligned}
\delta\left(T_{12}-T_{34}\right)= & 2 \frac{\overrightarrow{\boldsymbol{X}_{\mathrm{a}} \boldsymbol{X}_{\mathrm{a}}^{\prime}} \cdot \boldsymbol{v}_{\mathrm{g}}}{c^{2}}+\frac{\boldsymbol{D} \cdot \boldsymbol{\Delta} \boldsymbol{v}}{c \cdot D} \delta T_{23} \\
& +\left(\frac{\overrightarrow{\boldsymbol{X}_{\mathrm{a}} \boldsymbol{X}_{\mathrm{a}}^{\prime}} \cdot \boldsymbol{\Delta v}}{c \cdot D}-\frac{\boldsymbol{D}}{c \cdot D} \cdot \frac{\mathrm{d} \overrightarrow{\boldsymbol{X}_{\mathrm{a}} \boldsymbol{X}_{\mathrm{a}}^{\prime}}}{\mathrm{d} t}\right. \\
& \left.-\frac{\boldsymbol{D} \cdot \boldsymbol{\Delta} \boldsymbol{v}}{c \cdot D} \frac{\left\|\overrightarrow{\boldsymbol{X}_{\mathrm{a}} \boldsymbol{X}_{\mathrm{a}}^{\prime}}\right\|}{D}\right) T_{23} .
\end{aligned}
$$

We note that Eq. (17) depends the antenna phase center position error $\left(\overrightarrow{\boldsymbol{X}_{\mathrm{a}} \boldsymbol{X}_{\mathrm{a}}^{\prime}}\right)$, the coordinate time interval $T_{23}$ and the uncertainty on this time interval $\delta T_{23}$.

On the other hand, the clock relativistic correction along a trajectory is obtained from Eq. (8). It depends on the position and the velocity of the reference point, in this case the clock. We need to express the error in the reference point frequency - i.e., the frequency difference between the true clock position and the computed clock position - to compare its Modified Allan deviation with the specifications.

The gravitational potential can be evaluated on a given trajectory with sufficient precision (Wolf \& Petit 1995) using gravity models (e.g., GRIM5 or EGM96). The error in the frequency shift at the clock position is given by

$$
\begin{aligned}
\delta\left(\frac{\mathrm{d} \tau}{\mathrm{d} t}\right)_{X_{\mathrm{c}}}= & \left(\frac{\mathrm{d} \tau}{\mathrm{d} t}\right)_{X_{\mathrm{c}}}-\left(\frac{\mathrm{d} \tau}{\mathrm{d} t}\right)_{X_{\mathrm{c}}^{\prime}} \\
= & -\frac{1}{c^{2}}\left(U\left(t, \boldsymbol{X}_{\mathrm{c}}\right)-U\left(t, \boldsymbol{X}_{\mathrm{c}}^{\prime}\right)\right. \\
& \left.+\frac{V_{\mathrm{c}}^{2}-V_{\mathrm{c}}^{\prime 2}}{2}\right)
\end{aligned}
$$

The frequency difference between the reference point position $\boldsymbol{X}$ and the ISS center of mass $\boldsymbol{X}_{\mathrm{o}}$ is given by

$$
\begin{aligned}
\left(\frac{\mathrm{d} \tau}{\mathrm{d} t}\right)_{X}-\left(\frac{\mathrm{d} \tau}{\mathrm{d} t}\right)_{X_{\mathrm{o}}}= & -\frac{1}{c^{2}}\left(U(t, \boldsymbol{X})-U\left(t, \boldsymbol{X}_{\mathrm{o}}\right)\right. \\
& \left.+\frac{V^{2}-V_{\mathrm{o}}^{2}}{2}\right) .
\end{aligned}
$$

The trajectory of $\boldsymbol{X}_{\mathrm{o}}$ is the solution of the differential equation

$\frac{\mathrm{d}^{2} \boldsymbol{X}_{\mathrm{o}}}{\mathrm{d} t^{2}}=\boldsymbol{\Gamma}_{\mathrm{P}}+\boldsymbol{\Gamma}_{\mathrm{S}}$

where $\boldsymbol{\Gamma}_{\mathrm{P}}$ is the acceleration due to the gravitational potential and $\boldsymbol{\Gamma}_{\mathrm{S}}$ is the acceleration due to other effects (e.g., surface forces such as air drag and radiation pressure).

Using

$\boldsymbol{\Gamma}_{\mathrm{P}}=\operatorname{Grad}(U)$,

and to first order

$U(t, \boldsymbol{X})=U\left(t, \boldsymbol{X}_{\mathrm{o}}\right)+\boldsymbol{\Gamma}_{\mathrm{P}}\left(\boldsymbol{X}_{\mathrm{o}}\right) \cdot \overrightarrow{\boldsymbol{X}_{\mathrm{o}} \boldsymbol{X}}$,

and multiplying Eq. (20) by the vector $\overrightarrow{\boldsymbol{X}_{\mathrm{o}} \boldsymbol{X}}$ (which is the position of the clock with respect to the ISS center of mass), we can substitute the difference of gravitational potential in Eq. (19) by

$$
\begin{aligned}
U(t, \boldsymbol{X})-U\left(t, \boldsymbol{X}_{\mathrm{o}}\right) & =\boldsymbol{\Gamma}_{\mathrm{P}}\left(\boldsymbol{X}_{\mathrm{o}}\right) \cdot \overrightarrow{\boldsymbol{X}_{\mathrm{o}} \boldsymbol{X}} \\
& =\frac{\mathrm{d}^{2} \boldsymbol{X}_{\mathrm{o}}}{\mathrm{d} t^{2}} \cdot \overrightarrow{\boldsymbol{X}_{\mathrm{o}} \boldsymbol{X}}-\boldsymbol{\Gamma}_{\mathrm{S}} \cdot \overrightarrow{\boldsymbol{X}_{\mathrm{o}} \boldsymbol{X}}
\end{aligned}
$$




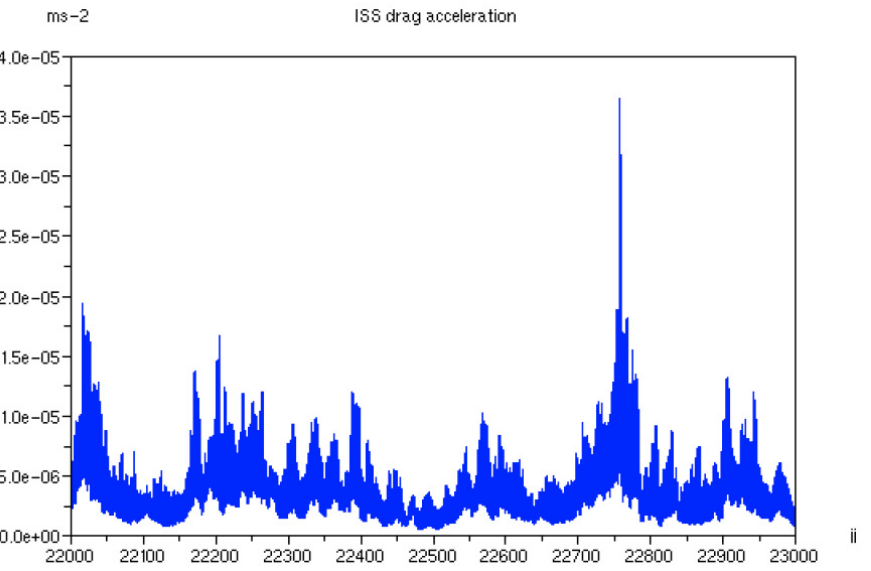

Fig. 2. Estimation of the non-gravitational acceleration of the ISS vs. time (in days)

We then obtain

$$
\begin{aligned}
\left(\frac{\mathrm{d} \tau}{\mathrm{d} t}\right)_{\boldsymbol{X}}-\left(\frac{\mathrm{d} \tau}{\mathrm{d} t}\right)_{\boldsymbol{X}_{\mathrm{o}}}= & -\frac{1}{c^{2}}\left(\frac{\mathrm{d} \boldsymbol{V}_{\mathrm{o}}}{\mathrm{d} t} \cdot \overrightarrow{\boldsymbol{X}_{\mathrm{o}} \boldsymbol{X}}+\boldsymbol{V}_{\mathrm{o}} \cdot \frac{\mathrm{d} \overrightarrow{\boldsymbol{X}_{\mathrm{o}} \boldsymbol{X}}}{\mathrm{d} t}\right. \\
& \left.+\frac{1}{2}\left(\frac{\mathrm{d} \overrightarrow{\boldsymbol{X}_{\mathrm{o}} \boldsymbol{X}}}{\mathrm{d} t}\right)^{2}-\boldsymbol{\Gamma}_{\mathrm{S}} \cdot \overrightarrow{\boldsymbol{X}_{\mathrm{o}} \boldsymbol{X}}\right),
\end{aligned}
$$

which can be simplified to

$$
\begin{aligned}
\left(\frac{\mathrm{d} \tau}{\mathrm{d} t}\right)_{X}-\left(\frac{\mathrm{d} \tau}{\mathrm{d} t}\right)_{X_{\mathrm{o}}}= & -\frac{1}{c^{2}}\left(\frac{\mathrm{d}}{\mathrm{d} t}\left(\boldsymbol{V}_{\mathrm{o}} \cdot \overrightarrow{\boldsymbol{X}_{\mathrm{o}} \boldsymbol{X}}\right)\right. \\
& \left.+\frac{1}{2}\left(\frac{\mathrm{d} \overrightarrow{\boldsymbol{X}_{\mathrm{o}} \boldsymbol{X}}}{\mathrm{d} t}\right)^{2}-\boldsymbol{\Gamma}_{\mathrm{S}} \cdot \overrightarrow{\boldsymbol{X}_{\mathrm{o}} \boldsymbol{X}}\right) .
\end{aligned}
$$

In this expression the term $\frac{1}{2}\left(\frac{\mathrm{d} \overrightarrow{\boldsymbol{X}_{\mathrm{o}} \boldsymbol{X}}}{\mathrm{d} t}\right)^{2}-\boldsymbol{\Gamma}_{\mathrm{S}} \cdot \overrightarrow{\boldsymbol{X}_{\mathrm{o}} \boldsymbol{X}}$ can be interpreted as the relativistic correction for the clock referenced to the local ISS frame. For an observer in the ISS frame, the nongravitational acceleration $\boldsymbol{\Gamma}_{\mathrm{S}}$ produces an acceleration that can be seen by this observer as coming from a "gravitational potential" $-\boldsymbol{\Gamma}_{\mathrm{S}} \cdot \overrightarrow{\boldsymbol{X}_{\mathrm{o}} \boldsymbol{X}}$. The term $\frac{\mathrm{d}}{\mathrm{d} t}\left(\boldsymbol{V}_{\mathrm{o}} \cdot \overrightarrow{\boldsymbol{X}_{\mathrm{O}} \boldsymbol{X}}\right)$ is the position of the clock with respect to the ISS center of mass projected onto the ISS velocity direction (along-track component). If there are no external accelerations, and if the velocity of $\boldsymbol{X}$ in the ISS frame remains small, only this term is present.

Combining the previous equation with the same expressed in $\boldsymbol{X}^{\prime}$, we obtain

$$
\begin{aligned}
\delta\left(\frac{\mathrm{d} \tau}{\mathrm{d} t}\right)_{\boldsymbol{X}_{\mathrm{c}}}= & \frac{1}{c^{2}}\left(\frac{\mathrm{d}}{\mathrm{d} t}\left(\boldsymbol{V}_{\mathrm{o}} \cdot \overrightarrow{\boldsymbol{X}_{\mathrm{c}} \boldsymbol{X}_{\mathrm{c}}^{\prime}}\right)+\frac{1}{2}\left(\frac{\mathrm{d} \overrightarrow{\boldsymbol{X}_{\mathrm{o}} \boldsymbol{X}_{\mathrm{c}}}}{\mathrm{d} t}\right)^{2}\right. \\
& \left.-\frac{1}{2}\left(\frac{\mathrm{d} \overrightarrow{\boldsymbol{X}_{\mathrm{o}} \boldsymbol{X}_{\mathrm{c}}^{\prime}}}{\mathrm{d} t}\right)^{2}-\boldsymbol{\Gamma}_{\mathrm{S}} \cdot \overrightarrow{\boldsymbol{X}_{\mathrm{c}} \boldsymbol{X}_{\mathrm{c}}^{\prime}}\right)
\end{aligned}
$$

In order to simplify Eq. (26), we evaluate the order of magnitude of the different contributors appearing in this equation.

To investigate the importance of the non-gravitational term $\frac{\boldsymbol{\Gamma}_{\mathrm{S}} \cdot \overrightarrow{\boldsymbol{X}_{\mathrm{c}} \boldsymbol{X}_{\mathrm{c}}^{\prime}}}{c^{2}}$, the drag was modeled along a reference orbit of the ISS. A period with important solar activity was chosen to evaluate the worst case (see Fig. 2).

To estimate its effect on Eq. (26), the acceleration was multiplied by a $10 \mathrm{~m}$ bias, by a 10 meter random noise, or by a
$10 \mathrm{~m}$ sinusoidal function at orbital period, corresponding to possible attitude and orbit error effects of the ISS. The Allan deviation remains below $10^{-21}$, which is totally negligible here. This term also has no effect on the frequency accuracy at the $10^{-16}$ level that ACES is designed to achieve. In addition, the residual term of the second order Doppler shift $\frac{1}{2 c^{2}}\left[\left(\frac{\mathrm{d} \overrightarrow{\boldsymbol{X}_{\mathrm{o}} \boldsymbol{X}_{\mathrm{c}}}}{\mathrm{d} t}\right)^{2}-\left(\frac{\mathrm{d} \overrightarrow{\boldsymbol{X}_{\mathrm{o}} \boldsymbol{X}_{\mathrm{c}}^{\prime}}}{\mathrm{d} t}\right)^{2}\right]$ must be computed with the GCRS trajectories. The order of magnitude of these terms can be evaluated as $\sim\left(a \delta a \Omega^{2} / c^{2}\right)$ with $\Omega$ the orbital angular frequency, $a=\left\|\overrightarrow{\boldsymbol{X}_{\mathrm{o}} \boldsymbol{X}_{\mathrm{c}}}\right\|$, and $\delta a$ its error. For $a \leq 100 \mathrm{~m}$ and $\delta a \leq 10 \mathrm{~m}$, this effect is also totally negligible.

The only important term in the performance evaluation is thus the along-track term $\frac{1}{c^{2}} \frac{\mathrm{d}}{\mathrm{d} t}\left(\boldsymbol{V}_{\mathrm{o}} \cdot \overrightarrow{\boldsymbol{X}_{\mathrm{c}} \boldsymbol{X}_{\mathrm{c}}^{\prime}}\right)$, and Eq. (26) can be written

$$
\begin{aligned}
\delta\left(\frac{\mathrm{d} \tau}{\mathrm{d} t}\right)_{\boldsymbol{X}_{\mathrm{c}}} & =\left(\frac{\mathrm{d} \tau}{\mathrm{d} t}\right)_{\boldsymbol{X}_{\mathrm{c}}}-\left(\frac{\mathrm{d} \tau}{\mathrm{d} t}\right)_{\boldsymbol{X}_{\mathrm{c}}^{\prime}} \\
& =\frac{1}{c^{2}}\left[\frac{\mathrm{d}}{\mathrm{d} t}\left(\boldsymbol{V}_{\mathrm{o}} \cdot \overrightarrow{\boldsymbol{X}_{\mathrm{c}} \boldsymbol{X}_{\mathrm{c}}^{\prime}}\right)\right]
\end{aligned}
$$

Therefore, only the component of the clock position error $\overrightarrow{\boldsymbol{X}_{\mathrm{c}} \boldsymbol{X}_{\mathrm{c}}^{\prime}}$ projected onto the velocity of the ISS $\boldsymbol{V}_{\text {o }}$ plays a role. This can be understood by considering for example a purely positive radial component. In this case, we underestimate the gravitational potential but overestimate the velocity, and the two effects cancel. We emphasize that the derivations in this chapter are valid for any type of orbital errors, up to the neglected terms described above.

The scalar products of vectors can be evaluated in a local frame. For example, it may be useful to study them in the local orbital frame $(\boldsymbol{R}, \boldsymbol{T}, \boldsymbol{N})$ defined by $\boldsymbol{R}$, which is the unit vector between the Earth's center and the space station, $N$, which is orthogonal to $\boldsymbol{R}$ and the inertial velocity, and $\boldsymbol{T}$, which is orthogonal to $\boldsymbol{R}$ and $\boldsymbol{N}$.

The ISS attitude is assumed to be roughly constant. The clock position error in this frame is represented by a constant vector (error in the position of the clock relative to the centre of mass), and by perturbations which produce

- attitude uncertainties (rigid body behaviour of ISS). If we take \pm 5 degrees uncertainty and a distance of 10 meters, this leads to a $0.87 \mathrm{~m}$ amplitude error in the clock position;

- ISS deformations: these are caused by thermoelastic effects, which will occur mainly at orbital period (eclipses and sun orientation). We suppose that these effects have an amplitude of below one meter;

- vibrations: here we assume that the "vibrations" are related to frequencies higher or equal to the first ISS eigenfrequency, which is higher than the orbital period. The corresponding displacements remain small and are expected to remain below one meter.

All of these effects are negligible when included into Eqs. (17) and (27). Therefore, for our purposes, the only significant contribution to the trajectory errors in both the antenna phase center and the clock come from the position errors of the ISS center of mass.

In summary, the errors in the time transfer and in the relativistic correction have been expressed as functions of the trajectory knowledge by means of Eqs. (17) and (27). Only the error in the knowledge of the ISS center of mass position plays a significant role in the relativistic correction. 


\section{Orbit error examples}

We now describe two simple models for ISS orbit errors as examples of investigating numerically the effect that they should have on ACES performance.

We consider two methods used to evaluate the orbit of a satellite. On the one hand, the dynamical method takes into account the equations of motion to estimate the trajectory, it fits the measured data with an orbit satisfying the principles of celestial mechanics, and estimates a number of parameters that provide the best fit trajectory. On the other hand, the kinematical method uses the measurements of the satellite position (e.g., GPS data) directly with no a priori assumption about the form of the orbit. In both cases, the resulting orbit errors are in general not easily described by a simple model, but for our purposes we use two examples that should approximately reflect the orders of magnitude of the main orbit error contributions.

For dynamical orbit determination, the differences between the true and computed trajectories of the ISS center of mass are expected to show specific structures. For example, an eccentricity error has no long-term effect, but periodic errors can be important, and the radial, along track, and velocity errors are all correlated. For weak eccentricity orbits, the difference between two real orbits is given by the Hill model (or the ClohessyWiltshire model), which is an expansion of the uncertainties relative to a reference circular orbit (Colombo 1986; Colombo et al. 2004). If we suppose that a set of parameters exists that perfectly describes the true orbit of the ISS, then this model is a first approximation to the errors in the center of mass between the computed and the true orbits.

For kinematical orbit determination, one expects little a priori correlation between the different orbital error components. We therefore use a simple independent random noise on each component as a first approximation.

In the Hill model, the position uncertainties along the radial, tangential, and normal axis (in the local ISS frame) are given as follows:

$$
\begin{aligned}
\text { radial axis: } \delta R(t) & =\frac{1}{2} X \cdot \cos \left(\Omega t+\varphi_{R}\right)+c_{R} \\
\text { tangential axis: } \delta T(t) & =-X \cdot \sin \left(\Omega t+\varphi_{R}\right)-\frac{3}{2} \Omega \cdot c_{R} \cdot t+d_{R} \\
\text { normal axis: } \delta N(t) & =Y \cdot \cos \left(\Omega t+\varphi_{N}\right)
\end{aligned}
$$

where $X, Y, c_{R}$, and $d_{R}$ are amplitude coefficients, and where $\Omega$ is the orbital angular frequency. For our purpose, the bias $\left(d_{R}\right)$ plays no role and the linear term $\left(c_{R}\right)$ depends on arc length. Basically, the longer the observation duration is, the smaller this coefficient becomes. The main feature of this error model is to take into account the error correlations in the orbital plane. For instance, a positive radial bias leads to a negative error in the tangential velocity: the satellite is delayed with respect to the reference orbit.

\section{Numerical results}

We now use the previously described error models to calculate the corresponding constraints imposed by the ACES stability (Eqs. (1) and (2)) and accuracy requirements, by means of Eqs. (17) and (27). We first consider the Hill model, followed by results for a simple random noise.

We consider orbit error models in the local $(\boldsymbol{R}, \boldsymbol{T}, \boldsymbol{N})$ frame, so we need to transform them to the GCRS and determine the uncertainties in this frame in position and velocity parameters. We consider an ephemeris of the ISS corresponding to the 20th

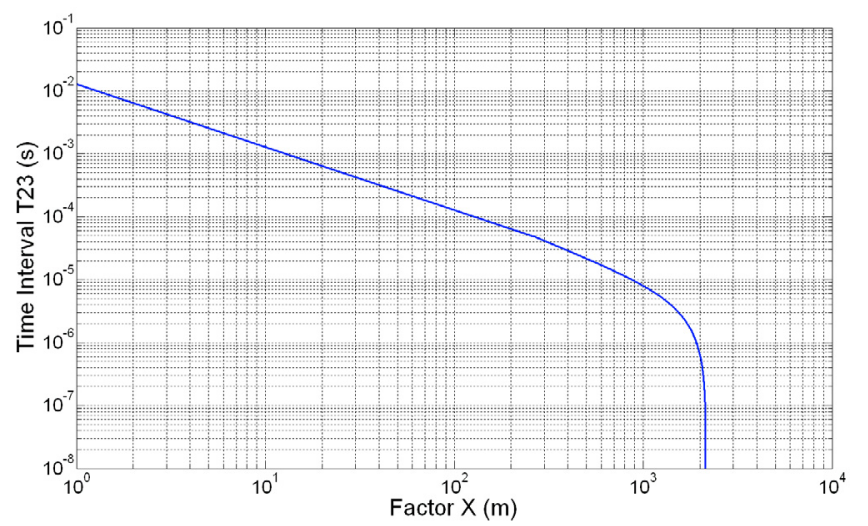

Fig. 3. The maximum allowed value of $T_{23}$ as a function of the scale factor $X$ to comply with the specifications, assuming $\delta T_{23}=0$.

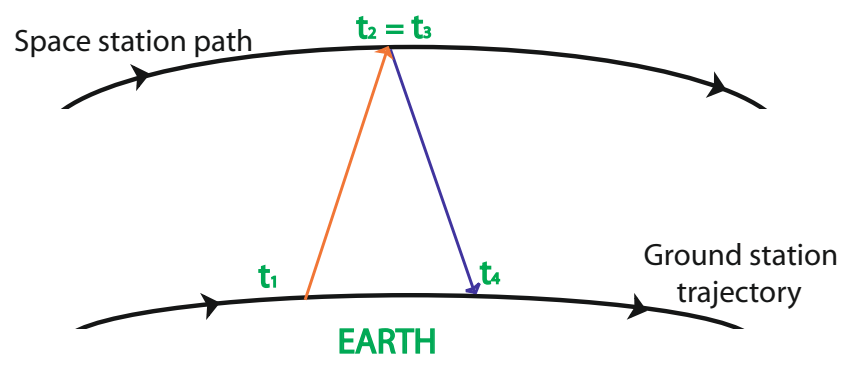

Fig. 4. The " $\Lambda$ configuration" is the way to combine upwards and downwards signals which allows the maximum uncertainties on the space station orbit determination.

of May, 2005 and a ground station based in Toulouse, France $\left(43^{\circ} 36^{\prime} N, 1^{\circ} 26^{\prime} E\right)$. This station has indeed been chosen as the master ground station of the ACES mission. All station parameters and their uncertainties have to be expressed in the same frame (i.e., GCRS).

We first consider the error in the time transfer given by Eq. (17). We choose the signs of the independent parameters $\left(\overrightarrow{\boldsymbol{X}_{\mathrm{a}} \boldsymbol{X}_{\mathrm{a}}^{\prime}}, T_{23}\right.$, and $\left.\delta T_{23}\right)$ so as to maximize the resulting temporal Allan deviation. The calculated deviation must be compared with the MWL specifications.

Assuming that the error in $T_{23}$ is negligible (i.e., $\delta T_{23}=0 \mathrm{~s}$ ), for all values of the factor $X$ (or $Y$ ) in Eq. (28), it is possible to determine the maximum value of the time interval $T_{23}$ for which the temporal Allan deviation remains smaller than the specifications. For numerous values of $X$, we calculate a bound that delineates two different areas: the allowed uncertainties area in which each couple of parameters $\left(X, T_{23}\right)$ gives a deviation staying under the specifications, and the prohibited area.

Figure 3 shows that, the shorter the time interval $T_{23}$, the larger the allowed uncertainty in the space station position. This result provides a way to combine upwards and downwards signals to allow the maximum uncertainty in space station position and to comply with the specifications. The most favorable situation is when the reception at the antenna phase center of the space station corresponds to the emission at the same place i.e., $t_{2}=t_{3}$ (see Fig. 4). This way of combining signals is named the " $\Lambda$ configuration". To work with parameters in the asymptotic area (see Fig. 3) we require $T_{23}$ to be below $10^{-6} \mathrm{~s}$.

If we then plot the maximum value of $\delta T_{23}$ for all values of the factor $X$, there will be two asymptotic values that we cannot cross if we want to stay within the specifications (see Fig. 5). 


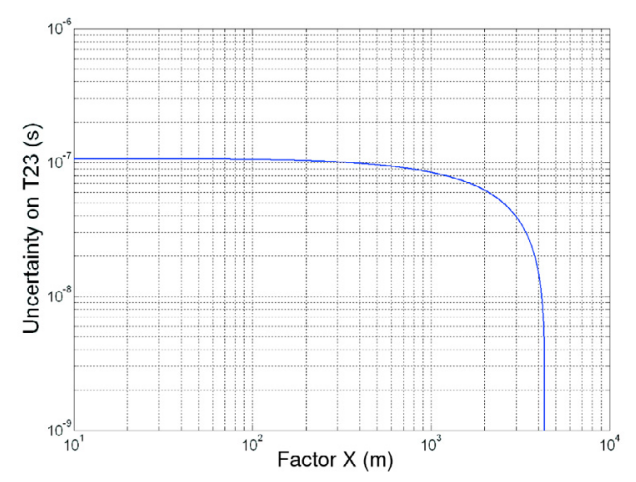

Fig. 5. Maximum allowed value of $\delta T_{23}$ as function of the scale factor $\mathrm{X}$ to comply with the specifications, assuming $T_{23}=0$.

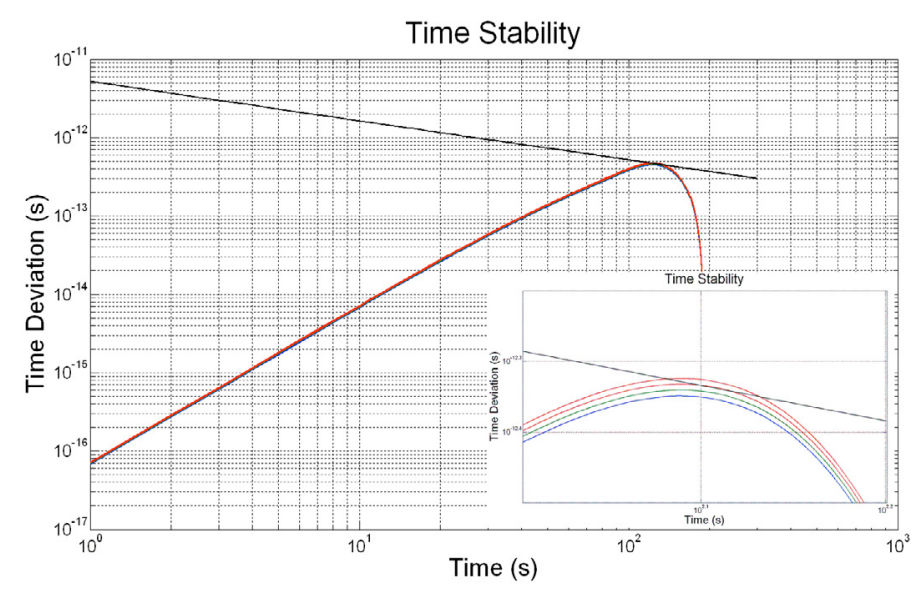

Fig. 6. Temporal Allan deviations for $X=0, T_{23}=0 \mathrm{~s}$ and $\delta T_{23}=$ $[102,104,106,108] \mathrm{ns}$.

Basically a compromise between the knowledge of the space station trajectory and the precision of the internal delay calibration must be achieved owing to the maximization of the Allan deviation. We will evaluate the maximum allowed errors in these two parameters if no other errors are present.

We search for the asymptotic value of factors $X$ and $Y$ that comply with the specifications for all phases $\left(\varphi_{R}, \varphi_{N}\right)$ when we have no error in $T_{23}$. The asymptotic value for the orbit determination is obtained for $X, Y=4200 \mathrm{~m}$, which corresponds to a $4.2 \mathrm{~km}$ error in the tangential and normal axes, and to a $2.1 \mathrm{~km}$ error in the radial axis.

The asymptotic value of the time calibration does not depend on the orbit determination uncertainties. So it is independent of the phases $\varphi_{R}$ and $\varphi_{N}$. We can then draw the temporal Allan deviations for different values of $\delta T_{23}$ (see Fig. 6). We find that $\delta T_{23}$ must stay below à $1.06 \times 10^{-7} \mathrm{~s}$.

The requirements for several passes have also been investigated. In this case, the calculated deviations have to be compared to the specifications given by Eq. (2). The results have shown that the requirements on orbit determination and time calibration are less stringent for several passes than for a single pass. Therefore, if specifications are respected for a single pass, specifications for longer integration times are also respected.

We now evaluate the requirements on orbit determination considering the relativistic frequency shift. We search for the maximum value of $X$ to comply with the specifications given by Eqs. (1) and (2). Equation (27) is evaluated with the error model in Eq. (28), and its Allan deviation is calculated for different

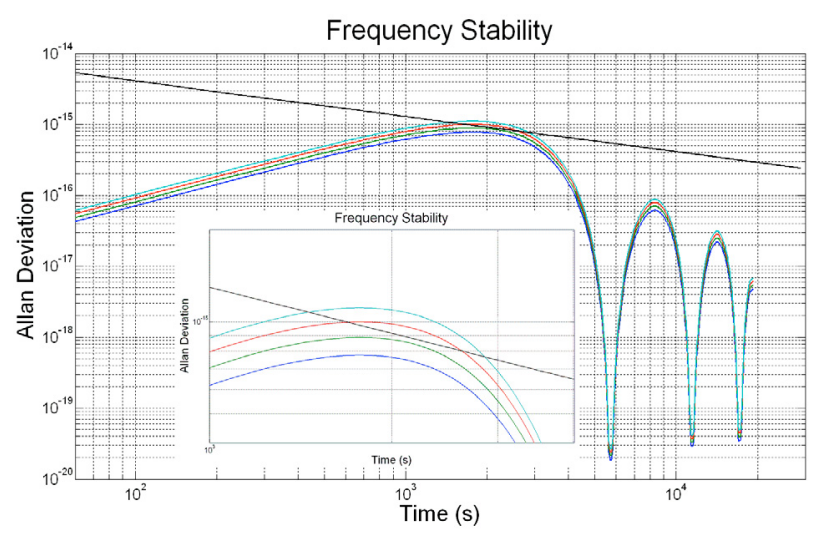

Fig. 7. Modified Allan deviations of the redshift error for $X=14,16$, $18,20 \mathrm{~m}$.

values of $X$. For integration times longer than one thousand seconds, these Allan deviations are independent of the phases $\varphi_{R}$ and $\varphi_{N}$.

Figure 7 shows that, if the factor $X$ in Eq. (28) equals $16 \mathrm{~m}$ i.e., if we have an eight meter error in the radial position and a sixteen meter error in the tangential position, then we comply with the specifications.

Because of the projection of the position error along the ISS center of mass velocity (see Eq. (27)), the requirement on the factor $Y$ is orders of magnitude less stringent. The bound is determined by the asymptotic value arising from the time transfer (see Eq. (17) and Fig. 5).

We now turn to the second example of orbit determination errors: independent random errors in $\boldsymbol{R}, \boldsymbol{T}$, and $\boldsymbol{N}$. We consider white noise of amplitude (standard deviation) $\delta R, \delta T$, and $\delta N$ at one second sampling intervals for each of the three components.

As for the Hill model, the by far more stringent constraints come from the effect of these errors in the relativistic frequency shift given by Eq. (27). In particular, a white noise $\delta T$ in Eq. (27) will translate into a temporal Allan variance given by

$\sigma_{x}(\tau) \simeq \frac{V_{0} \delta T}{c^{2}} \cdot \tau^{-\frac{1}{2}}$

which satisfies the requirements Eqs. (1) and (2) for all integration times when $\delta T \leq 60 \mathrm{~m}$.

Because of the projection of the error onto $V_{0}$ in Eq. (27), the relativistic frequency shift imposes virtually no limits on the normal and radial components of the errors. These are limited by the effect on the time transfer given by Eq. (17). Assuming that $T_{23} \leq 10^{-6} \mathrm{~s}$ and $\delta T_{23} \leq 10^{-7} \mathrm{~s}$ (see above), the first term of that equation is by far dominant. Maximizing the dot product in that term, we obtain an upper limit of $1.4 \mathrm{~km}$ to both $\delta R$ and $\delta N$ when remaining within specifications for all integration times.

Finally, we consider the accuracy requirement of ACES, i.e., $10^{-16}$ in relative frequency when averaged over ten days. From the integral of Eq. (27), this implies that the tangential component of the position error $\left(\overrightarrow{\boldsymbol{X}_{\mathrm{c}} \boldsymbol{X}_{\mathrm{c}}}\right)$ in Eq. (27)) cumulated over ten days needs to remain below one kilometer (including for example the linear term along the tangential axis in Eq. (28)). This is unlikely to raise any difficulty, if the far more stringent requirements from periodic or random errors (see above) are met. We note that the accuracy requirement is related to a requirement on the knowledge and conservation of the total energy of the orbit. An error in the total energy will show up as a velocity bias, and thus as a linear term along the tangential axis. The 
Table 1. Requirements on orbit determination and internal delay calibration.

\begin{tabular}{cccc}
\hline \hline$\delta R / \mathrm{m}$ & $\delta T / \mathrm{m}$ & $\delta N / \mathrm{m}$ & $\delta T_{23} / \mathrm{s}$ \\
\hline 8 & 16 & 1400 & $10^{-7}$ \\
\hline
\end{tabular}

non-conservation of the total energy (non-gravitational accelerations) was found to be negligible in Sect. 4. Nonetheless, possible long-term effects, if they exist, will lead to a slow variation in the tangential error. The constraint on both the error in the total energy and the effects due to energy non-conservation is provided by the accuracy requirement, i.e., that the tangential error cumulated over ten days needs to remain below one kilometer.

\section{Conclusion}

We have derived detailed and general (within the specified assumptions) expressions for the calculation of the effect of orbit determination and instrumental calibration errors, on the stability and accuracy of ground to space clock comparisons. These expressions were then used for the specific example of the ACES mission to derive the maximum allowed errors given the stability requirements of that mission. For that purpose, we used two simplified orbit error models (Hill model and random noise), setting limits on the appropriate parameters of those models. Although neither of those models is likely to reflect all of the ISS orbit errors correctly, they are nonetheless expected to provide reliable order of magnitude estimates of the maximum allowed orbit determination uncertainties. These are summarized in Table 1, where in each column we have provide the more stringent result of the two models.

Furthermore, we have identified an optimal way of combining upward and downward signals (the $\Lambda$ configuration), which allows for the maximum orbit determination errors. This then provides a constraint on the maximum allowed value of $T_{23} \leq 10^{-6} \mathrm{~s}$ and its maximum allowed uncertainty of $\delta T_{23} \leq$ $10^{-7} \mathrm{~s}$, which constrains the required knowledge of the sum (transmission + reception) of instrumental delays between the antenna phase centre and the onboard clock (also provided in Table 1).
Finally, the accuracy requirement of ACES was used to constrain long-term $(\approx 10$ days) linear drifts and other slow variations in the orbit determination errors. We find that the total tangential error cumulated over 10 days needs to remain below $1 \mathrm{~km}$ to comply with the accuracy specification $\left(10^{-16}\right.$ in relative frequency after ten days of averaging), which should pose no particular problems given the more stringent short-term requirements (see Table 1).

In conclusion, the requirements on the orbit determination are significantly less stringent than the initial 'naive' estimate (one meter error for $10^{-16}$ in relative frequency), which is mainly due to partial cancelation between the gravitational redshift and the second order Doppler effect in the relativistic frequency correction of the onboard clock.

Acknowledgements. The research project is supported by the French space agency CNES and ESA through Loïc Duchayne's research scholarship No. $05 / 0812$.

\section{References}

Allan, D. W., \& Ashby, N. 1986, in Relativity in Celestial Mechanics and Astronomy, ed. J., Kovalevsky, \& V. A., Brumberg, Proc. IAU Symp., 114, Leningrad 1985 (Dordrecht: Reidel Publ.)

Bahder, T. B. 2003, Phys. Rev. D, 68, 063005

Bassiri, S. \& Hajj, G. A. 1993, ISSN 0065-3438, 1071

Bize, S., Laurent, P., Abgrall, M., et al. 2005, J. Phys. B: At. Mol. Opt. Phys., 38, S449

Bjerhammar, A. 1985, Bull. Geod., 59, 207

Blanchet, L., Salomon, C., Teyssandier, P., Wolf, P., et al. 2001, A\&A, 370, 320

Colombo, O. L. 1986, Bulletin géodésique, 60, 64

Colombo, O. L., et al. 2004, Proceedings of the Institute Of Navigation GNSS Meeting

Duchayne, L. 2008, Ph.D. dissertation, in French, available on

http://tel.archives-ouvertes.fr/index . php

Heavner, T. P., Jefferts, S. R., Donley, E. A., Shirley, J. H., Parker, T. E., et al. 2005, Metrologia, 42, 411

Klioner, S. A. 1992, Celest. Mech. Dynam. Astron., 53, 81

Linet, B., \& Teyssandier, P. 2002, Phys. Rev. D, 66, 024045

Oskay, W. H., et al. 2006, Phys. Rev. Lett., 97, 020845

Petit, G., \& Wolf, P. 1994, A\&A, 286, 971

Rosenband, T., Hume, D. B., Schmidt, P. O., et al. 2008, Science, 319, 1808

Shapiro, I. I. 1964, Phys. Rev. Lett., 13, 789

Soffel, M. 2003, AJ, 126, 2687

Soffel, M., Rakuljic, G. A., Yariv, A., et al. 1988, Manuscripta Geodetica, 13143 Wolf, P., \& Petit, G. 1995 A\&A, 304, 653 